\title{
Unusual Semi-Spheric Perivesical Calcification after Pelvic Radiotherapy
}

\author{
Michael Froehner Oliver W. Hakenberg Andreas Manseck \\ Sven Oehlschlaeger Manfred P. Wirth \\ Department of Urology, University Clinic 'Carl Gustav Carus', Technical University of Dresden, Germany
}

\section{Key Words}

Calcification · Bladder · Pelvic · Radiotherapy

\begin{abstract}
An uncommon case with semi-spheric perivesical calcification after pelvic radiotherapy is reported and the possible pathogenesis of this phenomenon is discussed.
\end{abstract}

\section{Introduction}

Bladder wall calcification after pelvic radiotherapy is an uncommon phenomenon [1]. We report on a case with semi-spheric intrapelvic calcification probably involving the perivesical connective tissue rather than the detrusor muscle.

\section{Case Report}

A 63-year-old woman presented with post-renal acute renal failure due to chronic urine retention 27 years after Wertheim's radical hysterectomy with neoadjuvant and adjuvant radiotherapy for uterine cervix carcinoma. Abdominal plain X-ray films demonstrated a calciform intrapelvic calcification (fig. 1A). Intravenous urography

\begin{tabular}{ll}
\hline KARGER & ( 1999 S. Karger AG, Basel \\
Fax + 4161306 1234 & Ac42-1138/99/0622-0122\$17.50/0 \\
$\begin{array}{l}\text { E-Mail karger@karger.ch } \\
\text { www.karger.com }\end{array}$ & $\begin{array}{l}\text { Accessible online at: } \\
\text { http://BioMedNet.com/karger }\end{array}$
\end{tabular}

showed bladder contrast $1 \mathrm{~cm}$ apart from these calcified structures (fig. 1B). Voiding cystourethrography revealed a bilateral vesicoureteral reflux and long distal ureteral stenoses (fig. 2). A moderate radiation cystitis, a right-sided golf hole orifice and a marked bladder trabeculation were seen on cystoscopy. Urodynamics disclosed a hypertonic detrusor with instability and a high urinary residual volume. Frequent clean intermittent catheterization was recommended.

\section{Discussion}

Bladder wall calcification has been reported due to various causes such as bilharziosis, neoplasms, encrustation cystitis, tuberculosis, amyloidosis, cyclophosphamide, $\mathrm{BCG}$, mitomycin $\mathrm{C}$ and photodynamic therapy [2-6]. There are sparse reports on induction by radiotherapy [1, 2]. We identified only one similar case with radiationinduced vesical and paravesical calcifications [1]. In a second case, the post-irradiation bladder wall calcification was caused by concomitant alkaline encrusting cystitis and disappeared after irrigation of the bladder with an acid solution [7]. In the current case, calcification seemed to affect only the perivesical space. The semi-spheric shape of the calcification might be caused by impression of the dystrophic perivesical soft tissues by the chronically distended bladder. 

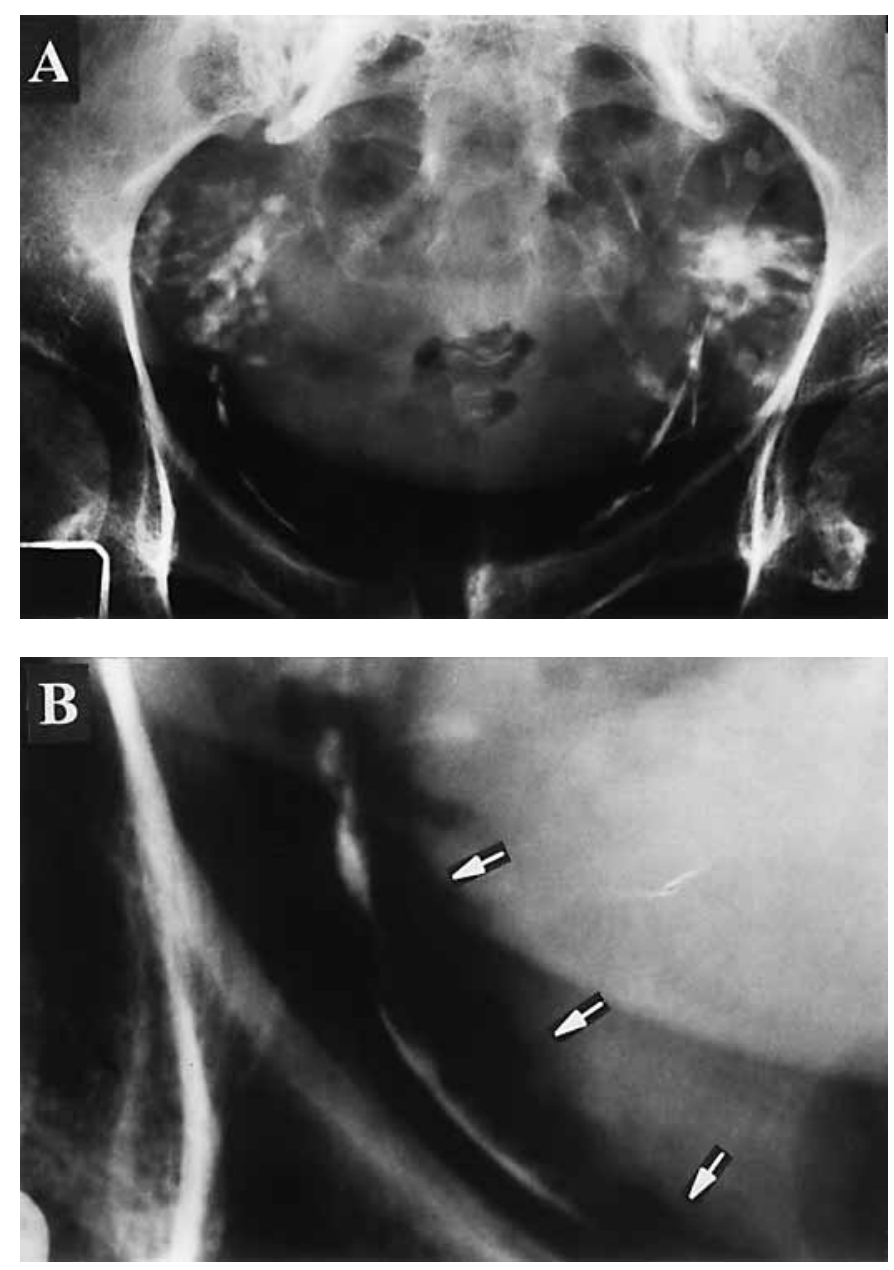

Fig. 1. A Semi-spheric perivesical calcification, abdominal plain Xray. B Intravenous pyelography demonstrating a bladder contrast about $1 \mathrm{~cm}$ from the califications (arrows).

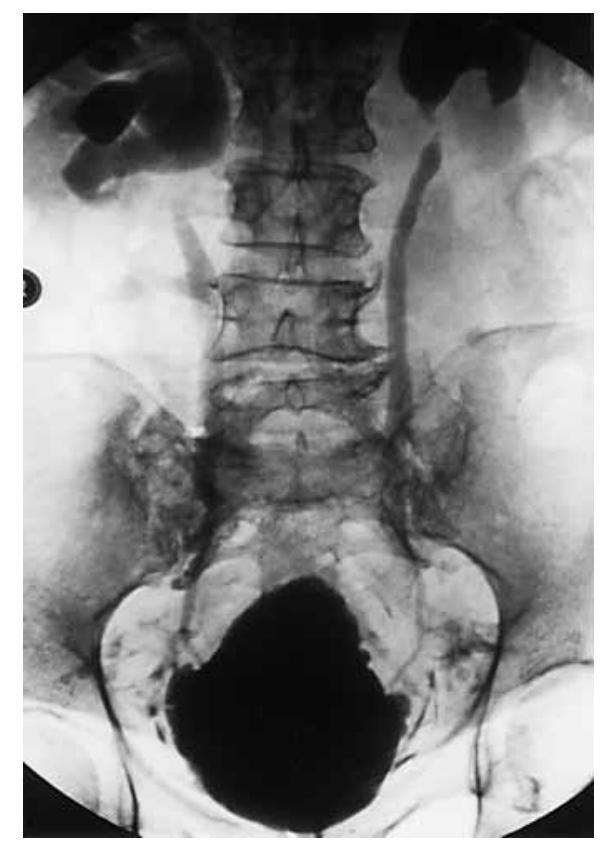

Fig. 2. Bilateral vesicoureteral reflux and long distal ureteral stenoses (voiding cystourethrography).

\section{References}

Unusual Perivesical Calcification
1 Grier DJ, Sibley GNA: A curious case of pelvic calcification. Br J Radiol 1992;65:945-946.

2 Pollack HM, Banner MP, Martinez LO, Hodson CJ: Diagnostic consideration in urinary bladder wall calcification. Am J Roentgenol 1981;136:791-797.

3 Spirnack JP, Lubke WL, Thompson IM, Lopez M: Dystrophic bladder wall calcification following BCG treatment for superficial transitional cell carcinoma of bladder. Urology 1993; 42:89-92.

4 Alter JA, Malek GH: Bladder wall calcification after topical mitomycin C. J Urol 1987;138: 1239-1240.
5 Drago PC, Badalament RA, Lucas J, Drago JR: Bladder wall calcification after intravesical mitomycin C of superficial bladder cancer. J Urol 1989;142:1071-1072.

6 Walther MM, Eanes ED, Delaney TF, Travis WD: Bladder calcification after photodynamic therapy: Analysis of a rare complication. Urology 1996;47:831-835.

7 Harrison BR, Stier FM, Cochrane JA: Alkaline encrusting cystitis. Am J Roentgenol 1978;130: 575-577. 